\title{
Comparación de la aceptabilidad de tres suplementos nutricionales
}

\author{
D. A. DE LUIS, G. CABEZAS, O. IZAOLA, R. ALLER ${ }^{1}$, L. CUÉLLAR, \\ M. C. TERROBA \\ Instituto de Endocrinología y Nutrición. Facultad de Medicina. Unidad de Investigación. \\ Hospital Río Hortega. ${ }^{~}$ Hospital Clinico. Universidad de Valladolid. Valladolid
}

\author{
COMPARATION OF SHORT TERM ACCEPTABILITY OF THREE \\ ORAL NUTRITIONAL SUPPLEMENTS
}

\section{RESUMEN}

Introducción: Aunque los suplementos nutricionales son fórmulas que aportan energía, minerales, vitaminas y proteínas, el consumo de estos productos en la mayor parte de las ocasiones depende de la aceptabilidad del paciente y de la habilidad para mantener consumos de volúmenes grandes a lo largo del tiempo.

Objetivos: Este estudio presenta como objetivo evaluar la aceptabilidad por parte de pacientes con tumores hematológicos de 3 suplementos nutricionales.

Diseño: Se estudiaron un total de 32 pacientes con tumores hematológicos y disminución de la ingesta (menos de un 50\% de los requerimientos calóricos determinados por la fórmula de Harrist Benedict). Los suplementos fueron asignados aleatoriamente a los pacientes, recibiendo el preparado durante dos semanas. Mediante una escala visual del 1 al 5 se evaluaron los siguientes parámetros (color, sabor, olor, textura, y temperatura).

Resultados: Un total de 10 pacientes recibieron el primer suplemento nuricional oral (SNO), 12 pacientes el segundo SON y 10 el tercero. No existieron diferencias epidemiológicas significativas entre los tres grupos. EL total de ingesta de calorías y macronutrientes mejoró con todos los suplementos. Los valores medios de color, sabor, olor, temperatura y textura fueron similares en los tres SON. Analizando mediante frecuencias relativas los resultados de la escala analógica, la frecuencia de las respuestas 1 y 2 (muy bueno y bueno, respectivamente) con respecto al sabor fue superior en el SON $2^{\circ}(85 \%$; p < 0,05$)$ que en el SON $1^{\circ}(50 \%)$ y que en SON $3^{\circ}(55,5 \%)$. Las frecuencias de las respuestas favorables (1 y 2$)$ con respecto al olor fueron superiores en el SON $2^{\circ}(83,3 \%$; p < $0,05)$ que en el primero $(55,5 \%)$ y en el tercero $(62,5 \%)$. Las frecuencias de las respuestas (bueno y muy bueno) fueron superiores en el primer SON $(90 \% ; \mathrm{p}<0,05)$ y en el segundo $(100 \% ; \mathrm{p}<0,05)$ que en el tercero $(66,7 \%)$. La valoración con respecto a la temperatura y color fueron similares en los tres suplementos.

Conclusiones: La aceptación de los diferentes suplementos orales nutricionales en los pacientes con tumores hematológicos es variable. Sabor, olor y textura fueron significativamente superior en algunos preparados.

PALABRAS CLAVE: Aceptabilidad. Pacientes. Suplementos orales.

\section{ABSTRACT}

Background: Although liquid supplements are formulated to provide extra energy, minerals, vitamins and proteins, much of the success of supplementation depends upon the acceptability of the product and the ability of the patients to take large volumes over a period of time.

Objective: This study was set up to evaluate the acceptability by haematological cancer patients of 3 commercially available nutritional supplements.

Design: A population of 32 haematological cancer patients with reduced food intakes (minor than 50\% of calory requirements by Harrist Benedict formula) was enrolled. Supplements were radomnly assigned to each patient. Patients could try the drinks over 2 days period. They were asked to rate the acceptability of supplement, rating the product with a visual scale from 1 to 5 points with five parameters (color, taste, smell, texture, and temperature).

Results: Ten patients received first ONS (oral nutritional supplements), 12 second ONS, and 10 third ONS. No epidemiological differences were detected among three groups. Total calorie and macronutrient consumption improved with all supplements. Average values of color, taste, smell, temperature and texture were similar on three oral nutritional supplements (ONS). Analogic scale was analyze in a categoric way with frequencies, too. Frequencies of 1 and 2 points (very good and good responses) with taste were better with second ONS $(85 \% ; p<0.05)$ than first ONS (50\%) and third ONS (55.5\%). Frequencies with smell were better with second ONS $(83.3 \% ; p<0.05)$ than first $(55.5 \%)$ and third ONS (62.5\%). Frequencies with texture were higher in first ONS (90\%; $p<0.05)$ and second ONS $(100 \% ; p<0.05)$ than third ONS (66.7\%). Temperature and color frequencies were similar in all ONS.

Conclusions: Oral nutritional supplements had different acceptability in haematological cancer patients. Taste, smell and texture could be better in some ONS in these patients.

KEY WORDS: Acceptability. Patients. Oral supplements.

de Luis DA, Cabezas G, Izaola O, Aller R, Cuéllar L, Terroba MC. Comparación de la aceptabilidad de tres suplementos nutricionales. An Med Interna (Madrid) 2007; 24: 15-18.

Trabajo aceptado: 8 de septiembre de 2006

Correspondencia: D. A. de Luis. Instituto de Endocrinología y Nutrición. Facultad de Medicina. Universidad de Valladolid. C/ Los Perales 16. (Urb. Las Aceñas). 47130 Simancas (Valladolid). 


\section{INTRODUCCIÓN}

Los pacientes tumorales presentan con frecuencia alteraciones en su situación nutricional. Estas alteraciones pueden ser secundarias a anorexia, aleraciones en el gasto energético y malabsorción. Algunos trabajos sugieren que la desnutrición calórico proteica aparece en estadios iniciales de los tumores $(1,2)$. La influencia del estado nutricional en el pronóstico de los pacientes tumorales se ha demostrado en diversos pacientes (3).

Habitualmente el soporte nutricional en este colectivo de pacientes se realiza con nutrición enteral o nutrición parenteral (4), pudiendo presentar ambas molestias para el paciente. Probablemente la forma menos agresiva para iniciar un soporte nutricional sean los suplementos orales nutricionales (SON). EL uso de estos preparados ha aumentado en nuestro medio, apareciendo recientemente consensos sobre el uso de estos productos en determinadas patologías (5).

Aunque los suplementos nutricionales son fórmulas que aportan energía, minerales, vitaminas y proteínas, el consumo de estos productos en la mayor parte de las ocasiones depende de la aceptabilidad del paciente y de la habilidad para mantener consumos de volúmenes grandes a lo largo del tiempo.

Este estudio presenta como objetivo evaluar la aceptabilidad por parte de pacientes con tumores hematológicos de 3 suplementos nutricionales.

\section{MATERIAL Y MÉTODOS}

\section{PACIENTES}

Se reclutaron un total de 32 pacientes con tumores hematológicos con disminución de la ingesta (menos de un 50\% de los requerimientos calóricos calculados mediante la fórmula de Harrist Benedict) (6). Los criterios de exclusión incluyeron; alteración de la función hepática severa (bilirrubina total $>3,5$ $\mathrm{mg} / \mathrm{dl}$ ), función renal alterada (creatinina sérica $>2,5 \mathrm{mg} / \mathrm{dl}$ ), infección intercurrente y consumo de medicación que pudiera alterar el consumo de alimentos y/o el peso corporal. El trabajo fue realizado de manera prospective desde mayo 2004 hasta diciembre del 2005. Los pacientes firmaron un consentimiento informado.

La evaluación basal de todos los pacientes consistió en la medición del peso, talla, cálculo de índice de masa corporal $\left(\mathrm{kg} / \mathrm{m}^{2}\right)$, circunferencia muscular del brazo, pliegue tricipital y un cuestionario dietético de 48 horas.

\section{SUPLEMENTOS Y ACEPTABILIDAD}

En las primeras 48 horas de ingreso hospitalario, los pacientes con tumores hematológicos con una ingesta calórica inferior al $50 \%$ de las necesidades energéticas calculadas con la fórmula de Harris Benedict recibieron dos unidades al día de un SON de manera aleatorizada. Los tres suplementos están comercializados en forma líquida y sus características nutricionales se presentan en la tabla I.

Tras la toma durante dos días del suplemento, se administró una escala visual de aceptabilidad para que valoraran del 1 al 5 los siguientes parámetros (color, sabor, olor, textura y temperatura). Se consideró la evaluación durante dos días para evitar interacciones con otras variables durante un ingreso hospitalario prolongado.

\section{TABLA I}

COMPOSICIÓN DE LOS SUPLEMENTOS POR 100 ML

\begin{tabular}{lccc}
\hline & Primer SON & Segundo SON & Tercer SON \\
\hline Volumen (ml) & 215 & 200 & 200 \\
Energía $(\mathrm{kcal})$ & 95 & 125 & 150 \\
Proteina $(\mathrm{g})$ & 5,3 & 6 & 4 \\
Lípidos $(\mathrm{g})$ & 2,7 & 3,2 & 3,9 \\
Carbohidratos $(\mathrm{g})$ & 13,8 & 12 & 12,3 \\
\hline
\end{tabular}

SON: suplemento oral nutricional.

\section{INGESTA DIETÉTICA}

Las ingestas de 48 horas fueron procesadas por una dietista utilizando un software propio de la Unidad con las tablas nacionales de composicón de alimentos (7). La ingesta total fue calculada teniendo en cuenta la ingesta de alimentos y los suplementos.

\section{VARIABLES ANTROPOMÉTRICAS}

En el inicio del estudio el peso y la talla de los pacientes fue medida $\left(\mathrm{Omron}^{\circledR}\right)$, calculándose el índice de masa corporal $\left(\mathrm{kg} / \mathrm{m}^{2}\right)$. También se midieron la circunferencia del brazo, el pliegue tricipital y se calculó la circunferencia muscular del brazo.

\section{DETERMINACIONES BIOQUÍMICAS}

En todos los pacientes se determinó en ayunas los niveles de albúmina $(3,5-4,5 \mathrm{~g} / \mathrm{dl})$, prealbúmina $(18-28 \mathrm{mg} / \mathrm{dl})$, transferrina $(250-350 \mathrm{mg} / \mathrm{dl})$ y linfocitos $\left(1,2-3,5.10^{3} / \mu \mathrm{L}\right)$ con un autoanalizador (Hitachi, ATM, Manheim, Ger).

\section{ESTADÍSTICA}

Los resultados se expresaron como media \pm desviación estandar. EL tamaño muestral fue calculado para detectar una diferencia del $25 \%$ con respecto a la mejores respuestas de aceptabilidad con un poder del $90 \%$ y una significación del $5 \%(\mathrm{n}=30,10$ pacientes en cada grupo). La distribución de las variables fue analizada con el test de Kolmogorov-Smirnov. Las variables cuantitativas con distribución normal se compararon mediante el test de la t de Student, y en los casos necesarios mediante ANOVA. Las variables cuantitativas no paramétricas fueron analizadas mediante los tests de Friedman y Wilcoxon. La correlación de Pearson se uso para determinar la relación entre diferentes variables. Un valor de $\mathrm{p}<$ 0,05 fue considerado como significativo.

\section{RESULTADOS}

Se reclutaron un total de 32 pacientes con una edad media de 47,6 \pm 16 años (19 mujeres/13 varones). Las características 
epidemiológicas de los pacientes se muestran en la tabla II. Un total de 10 pacientes recibieron el primer suplemento nutricional oral (SNO), 12 pacientes el segundo SON y 10 el tercero. No existieron diferencias epidemiológicas significativas entre los tres grupos.

\begin{tabular}{lc}
\multicolumn{2}{c}{ TABLA II } \\
\multicolumn{2}{c}{ PARÁMETROS ANTROPOMÉTRICOS } \\
\hline Edad (años) & $47,6 \pm 16,8$ \\
Hombre/mujer & $13 / 19$ \\
Peso (kg) & $68,2 \pm 12,8$ \\
IMC & $24,2 \pm 4,6$ \\
Pliegue tricipital (mm) & $16,6 \pm 8,9$ \\
Circunferencia muscular (cm) & $27,7 \pm 2,9$ \\
\hline
\end{tabular}

La tabla III muestra unos valores de albúmina, prealbúmina, transferrina y linfocitos inferiores a los de referencia, no existiendo diferencias entre los tres grupos (valores no mostrados).

TABLA III CARACTERÍSTICAS BIOQUÍMICAS

\begin{tabular}{lcc}
\hline Parámetros & \multicolumn{2}{c}{ Valores normales } \\
\hline Albúmina $(\mathrm{g} / \mathrm{dl})$ & $3,6 \pm 0,4$ & $(>3,5)$ \\
Prealbúmina $(\mathrm{mg} / \mathrm{dl})$ & $20,2 \pm 8,1$ & $(>25)$ \\
Transferrina $(\mathrm{mg} / \mathrm{dl})$ & $134 \pm 46,9$ & $(>200)$ \\
Linfocitos $\left(10^{3} \mathrm{uL} / \mathrm{mm}^{3}\right)$ & $657,1 \pm 316$ & $(>1.500)$ \\
\hline
\end{tabular}

Se administraron 2 unidades cada día de cada suplemento a cada paciente, alcanzándose un consumo promedio de 1,8 \pm 0,2 unidades/día. Con el primer SON, el consumo fue de $(1,7$ $\pm 0,1$ unidades/día), con el segundo suplemento (1,6 $\pm 0,4$ unidades/día) y con el tercero (1,9 $\pm 0,2$ unidades/day), sin diferencias significativas. El consumo calórico y de macronutrientes mejoró con los tres suplementos sin diferencias significativas entre los tres. En el análisis general de todos los pacientes se detectaron los siguientes valores: calorías: 1.322 $\pm 585 \mathrm{kcal} /$ día vs. $1.180 \pm 562 \mathrm{kcal} /$ día; $\mathrm{p}<0,05$; proteínas: $63,1 \pm 33$ g/día vs. 56,5 \pm 27,4 g/día; $\mathrm{p}<0,05$; carbohidratos: $160,9 \pm 71,4$ g/día vs. $147,1 \pm 68,8 \mathrm{~g} /$ día; $\mathrm{p}<0,05 ;$ y lípidos: 47,3 \pm 23 g/día vs. $43 \pm 23,4$ g/día; $\mathrm{p}<0,05$.

El valor medio de los diferentes parámetros analizados (color, sabor, olor, temperatura y textura) fue similar en los tres suplementos (Tabla IV). No se detectaron correlaciones entre estos valores y variables bioquímicas y antropométricas nutricionales.

Los resultados de la escala visual se analizaron mediante las frecuencias relativas de respuestas del 1 al 5 en cada parámetro analizado. Las frecuencias de las respuestas 1 y 2 (muy bueno y bueno, respectivamente) no mostraron diferencias con respecto a la evaluación del color; primer SON (90\%), segundo SON (91,7\%), y tercer SON (100\%). Las frecuencias de las respuestas 1 y 2 con respecto al sabor fueron mejores en el suplemento $2(85 \% ; \mathrm{p}<0,05)$ que en el primero $(50 \%)$ y en
TABLA IV

\begin{tabular}{lccc}
\multicolumn{4}{c}{ TABLA IV } \\
\multicolumn{4}{c}{ MEDIAS DE VARIABLES ORGANOLÉPTICAS DE } \\
ACEPTABILIDAD \\
\hline Parámetros & $2 \pm 0,4$ & $2,1 \pm 0,3$ & $1,8 \pm 0,3$ \\
\hline Color & $2,4 \pm 0,6$ & $2,1 \pm 0,5$ & $2,3 \pm 0,7$ \\
Sabor & $2,3 \pm 0,7$ & $2,1 \pm 0,4$ & $1,8 \pm 0,3$ \\
Olor & $2,1 \pm 0,3$ & $2 \pm 0,1$ & $2,3 \pm 0,8$ \\
Textura & $2,2 \pm 0,8$ & $2,1 \pm 0,5$ & $2,2 \pm 0,4$ \\
Temperatura &
\end{tabular}

Sin diferencias estadísticamente significativas; SON: suplemento oral nutricional.

el tercero $(55,5 \%)$. Con respecto al olor, fue superior el suplemento $2(83,3 \% ; \mathrm{p}<0,05)$ que el primero $(55,5 \%)$ y tercero $(62,5 \%)$. Las frecuencias sobre la textura fueron superiores con el primer SON $(90 \%$; p < 0,05) y con el segundo (100\%; p $<0,05)$ que con el tercer SON $(66,7 \%)$. El último parámetro organoléptico analizado (temperatura subjectiva) fue similar en todos los SON (primer SON 80\%, segundo SON 83,3\%, y tercero SON 77,8\%).

\section{DISCUSIÓN}

Los resultados de este estudio muestran cómo los SON son útiles para aumentar la ingesta calórica proteica en pacientes con cáncer hematológico. Las características organolépticas percibidas por los pacientes en las primeras 48 horas de ingesta son superiores en algunos de los suplementos del mercado.

La malnutrición es un problema importante en los pacientes con cáncer (1-3). Los suplementos pueden producir un incremento en la ingesta de pacientes con diferentes patologías. Nuestro grupo ha demostrado cómo en pacientes con infección por VIH se produce un mayor aumento del peso al utilizar suplementos orales frente al consejo nutricional aislado (8). Recientemente, Fearon y cols. (9) han demostrado cómo con suplementos enriquecidos en inmunonutrientes se puede aumentar el peso en un grupo de pacientes tumorales con muy mal pronóstico (cáncer de páncreas). No obstante este autor comenta que la aceptación por parte de los pacientes de estos suplementos es difícil, como también se ha demostrado con otros grupos de pacientes (10). Por tanto la aceptabilidad de los suplementos así como la capacidad para ingerir grandes volúmenes mantenidos durante el tiempo son puntos fundamentales en todos los protocolos de suplementación nutricional oral $(9,10)$.

La elección de un suplemento nutricional oral es realizada por el dietista, enfermera o médico responsable del paciente sin tener en cuenta las apetencias del paciente. Existiendo por otra parte poca evidencia en ese área de conocimiento. Diamond y cols. (11) han demostrado que existen clara diferencias entre la percepción que puede tener un dietista y la percepción de un paciente con respecto a un mismo suplemento oral. Mostrando diferencias significativas entre preparados comerciales de diferentes compañías.

Bolton y cols. (12) han mostrado que algunos suplementos orales nutricionales son más palatables que otros en pacientes tumorales, permitiendo mantener durante más días la suple- 
mentación oral. En los pacientes geriátricos (13), un suplemento nutricional específico para diabetes fue puntuado de manera superior que otro en las escalas de (apariencia, sabor, olor, cuerpo, regusto), a excepción de la dulzura.

Nuestro trabajo demuestra como con los tres suplementos la ingesta calórica proteica aumenta, en nuestro diseño de dos días. No obstante en un análisis de frecuencias el olor y el sabor fueron superiores en el segundo suplemento que en los otros dos, mostrándose la textura mejor en los dos primeros que en el tercero.

Nuestro trabajo presenta un diseño de evaluación a corto plazo y serán necesarios nuevos diseños para valorar como estas diferencias en la aceptación de determinados parámetros organolépticos pueden influir en la ingesta calóricoproteica de los pacientes. La situación de análisis se puede complicar si tenemos en cuenta que Gallagher y cols. (14) han mostrado como la aceptación de los suplementos varía con el tiempo.

En conclusión, la aceptación de los diferentes suplementos orales nutricionales en los pacientes con tumores hematológicos es variable. Sabor, olor y textura fue significativamente superior en algunos preparados.

\section{Bibliografía}

1. Lamisse F, May MA, Couet C, Constants T, Bacq Y, Delarue J, et al. Changes in nutritional status at the initial phase of treatment of cancers and malignant hemopathies. Rev Med Interne 1987; 8: 257-261.

2. Cederholm T, Eriksson K, Palmblad J. Nutrition and acute leukaemia in adults: relation to remission rate and survival. Haematologia 2002 ; 32: 405-417.

3. Alvim RC, Mesquita CJ, Cerqueira TA, Viana MB. Non-Hodgkin's lymphoma in children: analysis of nutritional status and other prognostic factors. J Pediatr 1996; 72: 400-410.

4. De Luis DA, Aller R, Izaola O, Cuéllar L, Terroba MC. Postsurgery enteral nutrition in head and neck cancer patients. Eur J Clin Nutr 2002; 56: 1126-1129.

5. Stratton RJ, ELia M. Are oral nutritional supplements of benefit to patients in the community? Findings from a systematic review. Curr Opin Clin Nutr Metab Care 2000; 3: 311-315.

6. Harris JA, Benedict FG. A biometric study of basal metabolism in man. Washington DC: Canergie Institute of Washington; 1919. p. 4044.

7. Mataix J, Mañas M. Tablas de composición de alimentos españoles. $3^{\mathrm{a}}$ ed. Ed: University of Granada; 1998.

8. De Luis DA, Aller R, Bachiller P, González Sagrado M, de Luis J, Izaola O. Consejo nutricional aislado frente a suplemento y consejos

nutricionales en pacientes con infección por VIH. Med Clin 2003; 120: $565-567$

9. Fearon KC, Von Meyenfeldt MF, Moses AG, Van Geenen R, Roy A, Gouma DJ. Effect of a protein and energy dense N3 fatty acid enriched oral supplement on loss of weight and lean tissue in cancer cachexia: a randomised double blind trial. Gut 2003; 52: 1479-1486.

10. De Luis DA, Aller R, Izaola O, Cuellar L, Terroba MC. Influence of a w3 fatty acids oral enhanced formula in clinical and biochemical parameters of head and neck cancer ambulatory patients. M Clin 2004; 123: 499-500.

11. Diamond L, Soon E. Sensory evaluation of oral nutritional supplements: a comparison of patients and dietitian preferences. J Can Diet Assoc 1994; 55: 85-90.

12. Bolton J, Shannon L, Smith V, Abbott R, Bell SJ, Stubbs L. Comparison of short term and long term palatability of six commercially available oral supplements. J Human Nutrit Diet 1990; 3: 317-321.

13. Grau T. Almazán JA, Luna A, Chamorro Quiros J, Lord Rodríguez T, Casimiro C. Evaulation of palatability of two special oral diets for institutionalized elderly diabetics, Glucerna SR vs Resource Diabet. Nutr Hosp 2004; 19: 292-299.

14. Gallagher P, Tweadle DE. Taste threshold and acceptability of commercial diets in cancer patients. J Parent Ent Nutr 1983; 7: 361-363. 